\title{
Aperçu \\ des théories mathématiques de la houle et nécessité de recherches expérimentales
}

\author{
Notes on mathematical wave theories \\ and the necessity of experimental research
}

\author{
i'ar M. le Professeur Dr. Ing. Luigi Greco

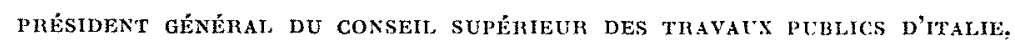 \\ PROFESSEUR EN TITRE DE CONSTRUCTIONS MARITHES A L'UNIVERSITE DE RONIE
}

\begin{abstract}
Dans une première partie, l'auteur donne un apercu des théories de la houle marine, en commençant par les études de Léonard de Vinci pour arriver $\dot{\alpha}$ celles des mathématiciens, des océanographes et des ingénieurs maritimes de nos jours. L'auteur se réfère également aux expériences de laboratoire sur modèles d̀ échelle réduite et aux expériences menées dans les slations expérimentales in situ et dans diners centres d'étude internationaux. Les insfallations dynomaréométriques et stéréophotogrammétriques du port de Naples font l'objet d'une description détaillée.
\end{abstract}

\begin{abstract}
In this first parl, the author gives us a glimpse of the theories of sea wates starling with Leonardo da Vinci's work and leading up lo the investigations of modern malhematicians, oceanographers and marilime engineers. The author also refers to laboratory experiments on scale models and to experiments carried out at experimental stations on site and al various international research centres. He gives " detaled description of wave strength measurement and stereo-phologrammetric installations at Naples harbour.
\end{abstract}

En Italie, le premier qui ait entrevu, par son génie, le mécanisme réel de la houle marine fut Léonard de Vinci. Dans son livre d'or: « Du mouvement et de la mesure de l'eau $\gg(1)$, par d'heureuses images agrestes, il a le premier affirmé que, dans les ondes d'oscillation du large soulevées par les vents marins, il n'y a pas de translation de masses mais seulement propagation de mouvement. Voici d'ailleurs comment il s'exprime : «Il arrive très souvent que la vague fuit le lieu de sa création, mais sans que l'eau change de place. Ne voit-on pas de même au mois de mai courir par les campagnes l'ondulation que fait le vent dans les blés, et pourtant les blés n'ont pas changé de place.»
La nature oscillatoire de la houle du large est exprimée d'une autre façon par Vinci quand il traduit ce phénomène par une autre image heureuse 《plutôt tremblement que mouvement». Et le livre d'or déjà cité contient de nombreuses considérations sur la houle, dans toules ses manifestations depuis le large jusqu'à la côte.

Dans les études bathylithologiques et psammographiques relatives au littoral et à la plateforme sous-marine qui le devance, Léonard de Vinci nous a également apporté le précieux tribut de son génie (2). Sur ce sujet, il convient de signaler les études du comte Luigi Ferdinando Marsili, de Cialdi, Rovereto, Cornaglia, Uzielli, Chelussi, Clerici, De Marchi, Issel, 
Possenti et d'autres, ainsi que plus récemment, les publications de d'Arrigo (3).

Par la suite, nous devons à Paolo Cornaglia, inspecteur du Corps Royal du Génie Civil italien, et ancien élève de l'Ecole Nationale des Ponts et Chaussées de Paris, un développement théorique triès original sur le flot de fond et sur les plages, çui a pu conduire à l'évaluation mathématique de l'action dynamique des lames contre les ouvrages à la mer, et à la concep- tion géniale de la ligne neutre des plages; c'est ce dernier concept qui a permis de repérer et d'analyser le mouvement des matériaux le long des rivages et perpendiculairement à eux, et qui a toujours fourni aux ingénieurs de travaux maritimes une grande contribution de connaissances précieuses (4).

La théorie de Cornaglia, comme toutes les théories qui entreprirent avec hardiesse de traduire les phénomènes naturels en des analyses mathématiques complexes, a été sujette à des critiques plus ou moins sereines. Pourtant, cette théorie, une fois approfondie et appliquée de façon pratique, offre certaines possibilités de calcul pour les structures d'ouvrages maritimes à la mer (jetées ou digues); nous l'avons ainsi appliquée pour le projet de la digue Duc des Abruzzes dans le port de Naples, comme on peut le relever sur un mémoire que nous avons publié dans les Annali dei LL. PO. (5).

C'est sur d'autres bases qu'un ingénieur de Prague, Franz von Gerstner, a imaginé la théorie trochoïdale des ondes d'oscillation (6), ellemême conçue génialement et réservant des possibilités analogues pour le calcul des ouvrages à la mer, grâce à l'extension de la théorie aux ondes qui se propagent en des profondeurs inférieures à leur longueur, ainsi que l'ont fait Sir George Airy (7) et Boussinesq (8) en s'aidant du principe de Gaillard (9) : selon ce critère, le flot qui rencontre un obstacle serait animé d'une vitesse égale à la somme de la vitesse de propagation de la houle et de la composante horizontale maximum de la vitesse orbitale animant les particules superficielles.

Comme ce contrôle s'imposait, nous n'avons pas manqué d'appliquer cette théorie à la digue Duc des Abruzzes du Port de Naples, avec des résultats également favorables (10).

De même que celle de Cornaglia, la théorie de Gerstner fut également sujette à des critiques (11); bien plus, Stokes l'a trouvée affectée d'inexactitude mathématique, en ce que le vecteur rotationnel y prend une valeur différente de zéro, alors que, suivant les principes de la mécanique classique, énoncés par Lagrange et par Helmoltz, le mouvement devrait être irrotationnel, puisqu'il concerne un liquide incompressible à frottements négligeables, parti de l'état de repos, et soumis à des forces ayant un potentiel de vitesse. Au lieu de cela, Stokes a démontré qu'en un point quelconque de l'oride étudiée par Gerstner, le vecteur rotationnel a une valeur définie différente de zéro.

Stokes a fourni au problème une solution approchée; une solution plus poussée a été établie par Lord Rayleigh, mais la solution mathématique rigoureuse pour les ondes d'oscillation irrotationnelles fut étudiée par notre éminent mathématicien Levi-Civita dans un célèbre mémoire, paru en 1925 dans les Mathematische Annalen.

Parmi les résultats les plus notables que ces recherches aient révélés, il convient de citer celui-là; avec la théorie de Gerstner, la vitesse de propagation de la houle est donnée par la formule :

$$
V=\sqrt{\frac{g \mathrm{~L}}{2 \pi}}
$$

L étant la longueur d'onde; d'après cette formule, $V$ ne dépend donc pas de la hauteur, alors qu'avec la théorie de Stokes sur le mouvement irrotationnel approché, on aboutit à une valeur de la vitesse de propagation par la formule :

$$
\mathrm{V}==\sqrt{\frac{g \mathrm{~L}}{2 \pi}} \sqrt{1+\alpha^{2}}
$$

arec :

$$
\alpha=\frac{2 \pi h}{\mathrm{~L}}
$$

$2 h$ étant la hauteur de la houle; avec la théorie rigoureuse de Levi-Civita, on obtient:

$$
\mathrm{V}=\sqrt{\frac{g \mathrm{~L}}{2 \pi}} \sqrt{1+\alpha^{2}-\alpha^{3}+\frac{7}{4} x^{4}-\frac{49}{12} \alpha^{5}+\ldots}
$$

étant toujours $\alpha=(2 \pi h) / \mathrm{L}$.

Ainsi, l'expression de la vitesse de propagation pour les houles irrotationnelles dépend donc également de la hauteur.

Par ailleurs, tandis que dans la théorie de Gerstner, les orbites se présentent comme des courbes fermées, dans les théories irrotationnelles, les orbites sont ouvertes, avec transport de liquide dans le sens de la propagation de la houle.

Ces théories traitent des manifestations de la houle en profondeur infinie; pour une houle se propageant en profondeur limitée, il convient de se reporter aux théories corrélatives de Airy, Stokes, Boussinesq, Sainflou, Struick, ete.

Une analyse mathématique approfondie et étendue des divers types de houle, en relation avec les différentes théories citées, a été conduite 
également par Bouasse dans une de ses imporlantes publications (12).

Les aspects assez changeants du mécanisme cinématique et dynamique de la houle en relation avec la configuration des parois externes des ouvrages rencontrés, ont conduit à une succession d'études importantes et vraiment estimables, à savoir : du point de vue statique, une étude sur le clapotis (onde systatique) de Sainflou et de Bénézit, du point de vue dynamostatique les études de Gourret, Miche et Lira, et les études de Cornaglia, Gaillard et Molitor du point de vue particulièrement dynamique.

En présence d'une si riche moisson d'études théoriques fondées sur des développements mathématiques élevés et parfois même géniaux, bien que non toujours concordants et décisifs, les ingénieurs maritimes, poussés par les exigences constructives, ont tenu à contrôler expérimentalement l'évaluation des actions dynamiques de la houle par des mesures directes déduites d'installations in situ (stations dynomaréométriques), ainsi que par des expériences sur « modèles à échelle réduite ».

C'est ainsi qu'ont vu le jour en Italie, la station dynomaréométrique du port de Naples (13) endommagée par suite de la guerre, puis reconstruite et complétée par une station stéréophotogrammétrique, et celle du port de Gênes, irrémédiablement détruite par la guerre et malheureusement non reconstruite (14).

A l'étranger, des installations de ce genre furent égalemènt créées : à commencer par le milieu du siècle précédent, Stevenson a installé en Ecosse et en Angleterre ses dynomaréomètres à ressorts; plus récemment, des installations in situ furent montées en France à Dieppe et au Havre, en Allemagne à Héligoland, en Algérie à la digue Mustapha du port d'Alger, en Russie à Touapsé sur la mer Noire et à Léningrad sur la Baltique, aux Etats-Unis sur le Lac Supérieur, à North Beach et à Saint-Augustin, au Japon à Otaru, Rumoi et au Cap Taito; les résultats ainsi obtenus permettent de déduire des éléments expérimentaux directs sur le phénomène de la houle, au droit des ouvrages rencontrés.

En ce qui concerne les expériences sur modèle à ćchelle réduite utilisant le principe de la similitude mécanique, il existe en Italie les laboratoires des Instituts universitaires d'hydraulique de Milan, de Padoue, de Rome et de Naples, et à l'étranger : les laboratoires de Chatou, de Maisons-Alfort et de Grenoble en France; de Delft en Hollande; ceux de Lausanne et de Zurich en Suisse; celui de l'Ecole d'Ingénieurs des routes, des canaux et des ports à Madrid en Espagne; celui de Hanove en Allemagne, elc. Tous entreprennent des recherches toujours plus importantes el plus instructives sur les phénomènes dynamiques de la mer, en relation avec les modalités d'installation et de construction des ouvrages des ports et fluviomaritimes. Ont fait l'objet d'une attention particulière le phénomène du mascaret et l'aménagement du tronçon maritime des fleuves qui débouchent en estuaire dans des mers à grand marnage, ce qui nous donne l'occasion de mentionner les modèles de la Seine de Maisons-Alfort et de Grenoble, et celui de l'estuaire de l'Elbe à Hambourg.

A propos des études et des observations concernant la morphologie de la houle, nous avons installé à Naples une station stércophotogrammétrique synchronisce avec une station dynomaréométrique. Il est alors possible de relever les caractéristiques géométriques de la houle du large, Iorsqu'elle arrive à proximité des ouvrages à la mer, et d'en déterminer les effets dynamiques qui sont transmis à des dynamomètres (capteurs) et enregistrés sur un oscillographe.

Ce remarquable ensemble d'appareils, doté de tous les perfectionnemnts, mis en place dans la nature et dans les laboratoires scientifiques, est là pour témoigner quels progrès ont marqué, au cours des cinquante dernières années, les études de hase sur la houle dans ses diverses formes.

\section{LES INSTALLATIONS DYNOMAREOMETRIQUES ET STÉREOPHOTOGRAMMETRIQUES DU PORT DE NAPLES}

Les premiers ouvrages dynomaréométriques du port de Naples, qui remontent à 1934, furent réalisés en installant un caisson expérimental dans le corps de l'infrastructure à paroi verticale de la digue Duc des Abruzzes (13), alors en construction.

L'extension à diverses cotes de la station d'enregistrement de la pression de la houle fut effectuée graduellement, en fonction des possi- bilités financières, l'appareillage oscillographique ayant été mis en place dès le début en vue de l'insertion graduelle des divers récepteurs.

La guerre, qui a causé tant de ruines au port de Naples, n'a pas épargné cette installation, qui avait déjà fourni quelques résultats appréciables dans sa phase d'expérience ef de mise au point.

Lorsque la conviction fut faile que les éludes 


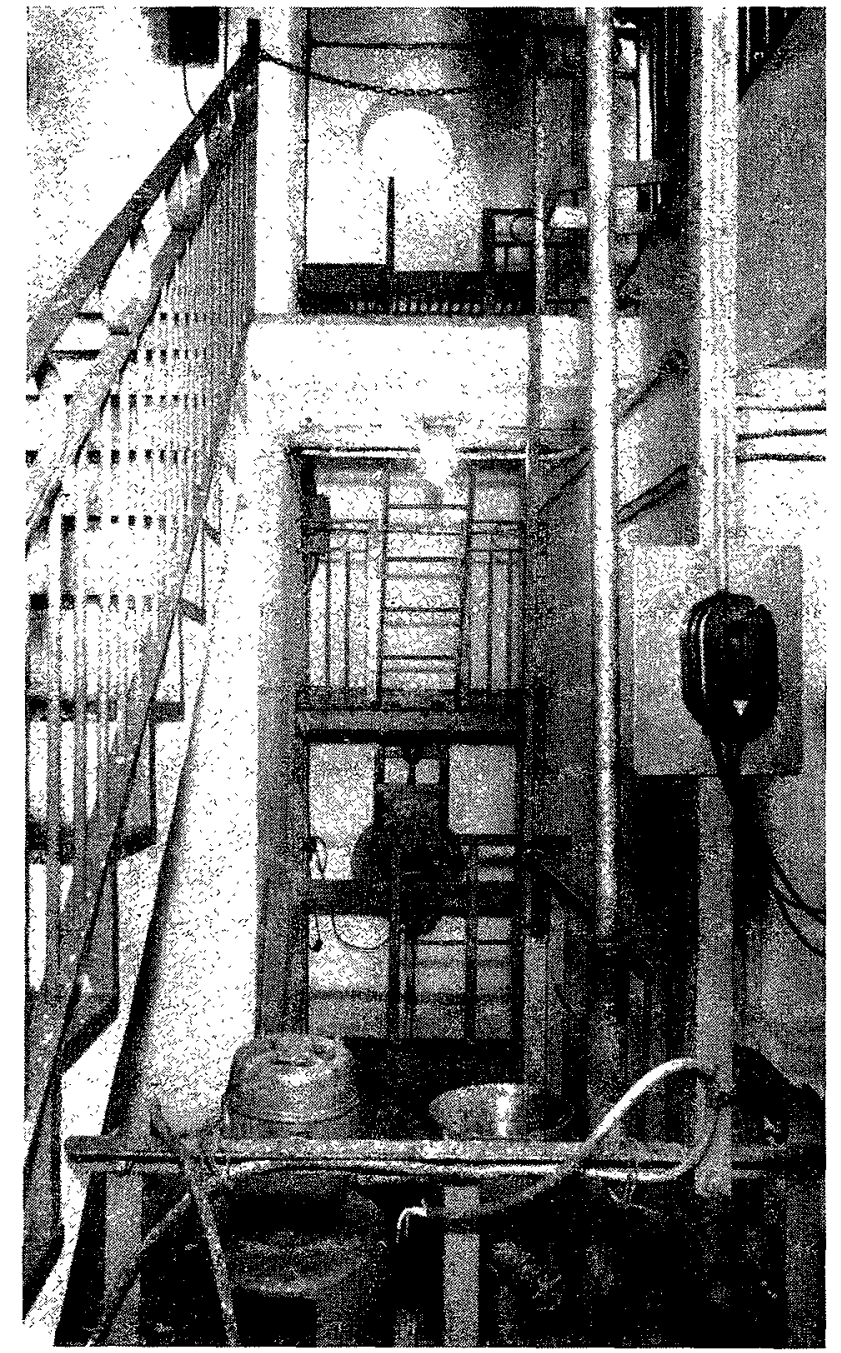

Photo A. - Station DyNomaréométrioue. Caisson expérimental avec capteur de houle. de la houle, el surtout des effets dynamiques qu'elle produit, doivent reposer essentiellement sur l'expérience, on dut considérer qu'il était d'un grand inlérêt de reconstruire la station primitive, non seulement en améliorant et en perfectionnant son appareillage, mais en y adjoignant une stalion de rolévements stéréophotogrammétriques capable de donner les éléments caractéristiques de la houle; or, ces éléments sont indispensables pour établir une relation entre les effets dynamiques de la houle et ses paramètres changeants.

\section{DESCRIPTION DES INSTALLATIONS}

Un plan d'ensemble des ouvrages est donné par la figure 1.

En particulier, les installations dynomaréométriques comprennent :

a) une cabine de relèvements sur la digue Duc des Abruzzes;

b) une cabine d'enregistrement sur la jetée Saint-Vincent, reliée ćlectriquement à la précédente.

Un caisson cellulaire en béton armé a éló inséré dans le corps de la digue Duc des Abruzzes, qui est formée par trois ordres de blocs cyclopéens, reposant sur une assise en enrochements. Dans la paroi frontale de ce caisson (voir fig. $2 a$ et $b$ ) et en correspondance avec un premier puits vertical, ont été pratiquées une série d'ouvertures circulaires qui servent de sicges aux plaques d'enregistrement, et ceci anx cotes

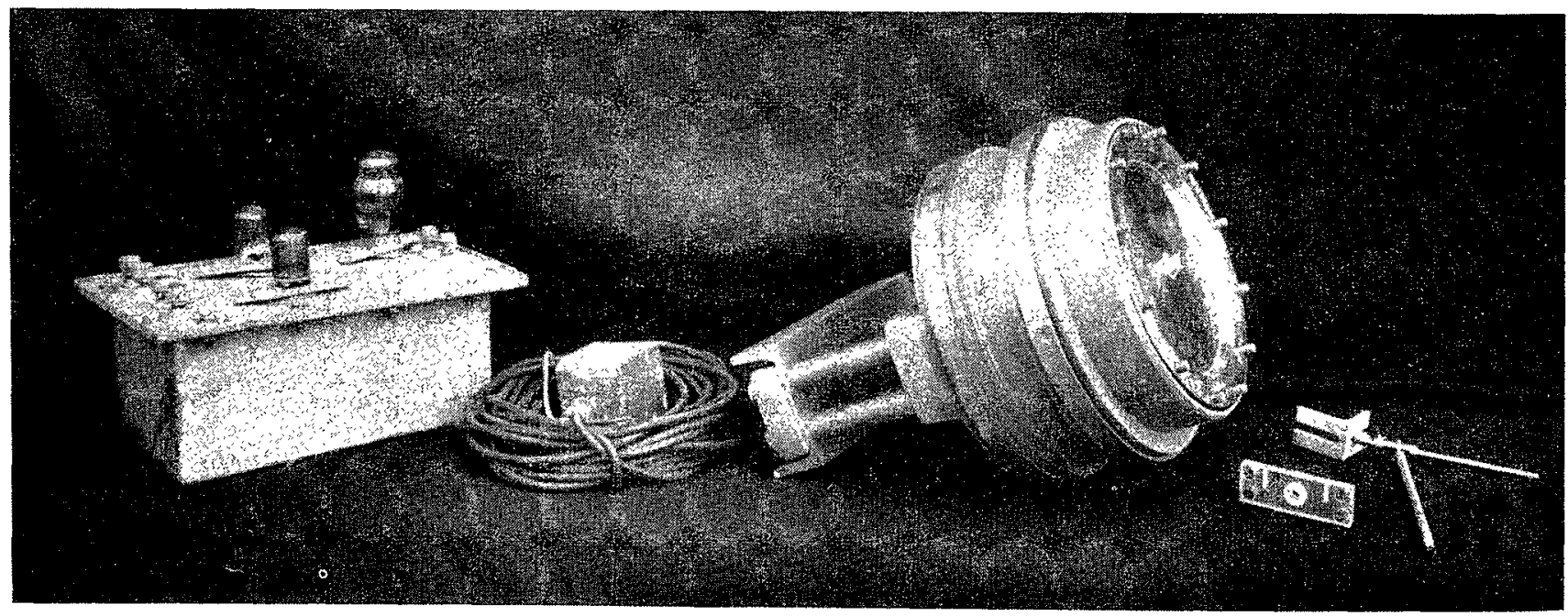

Photo B. - Station dynomaréométrique de Napress. Ensemble pour l'enregistrement de la pression de la houle, comprenant : oseillateur, cylindre capteur avec plaques, levier multiplicateur, inductance. 


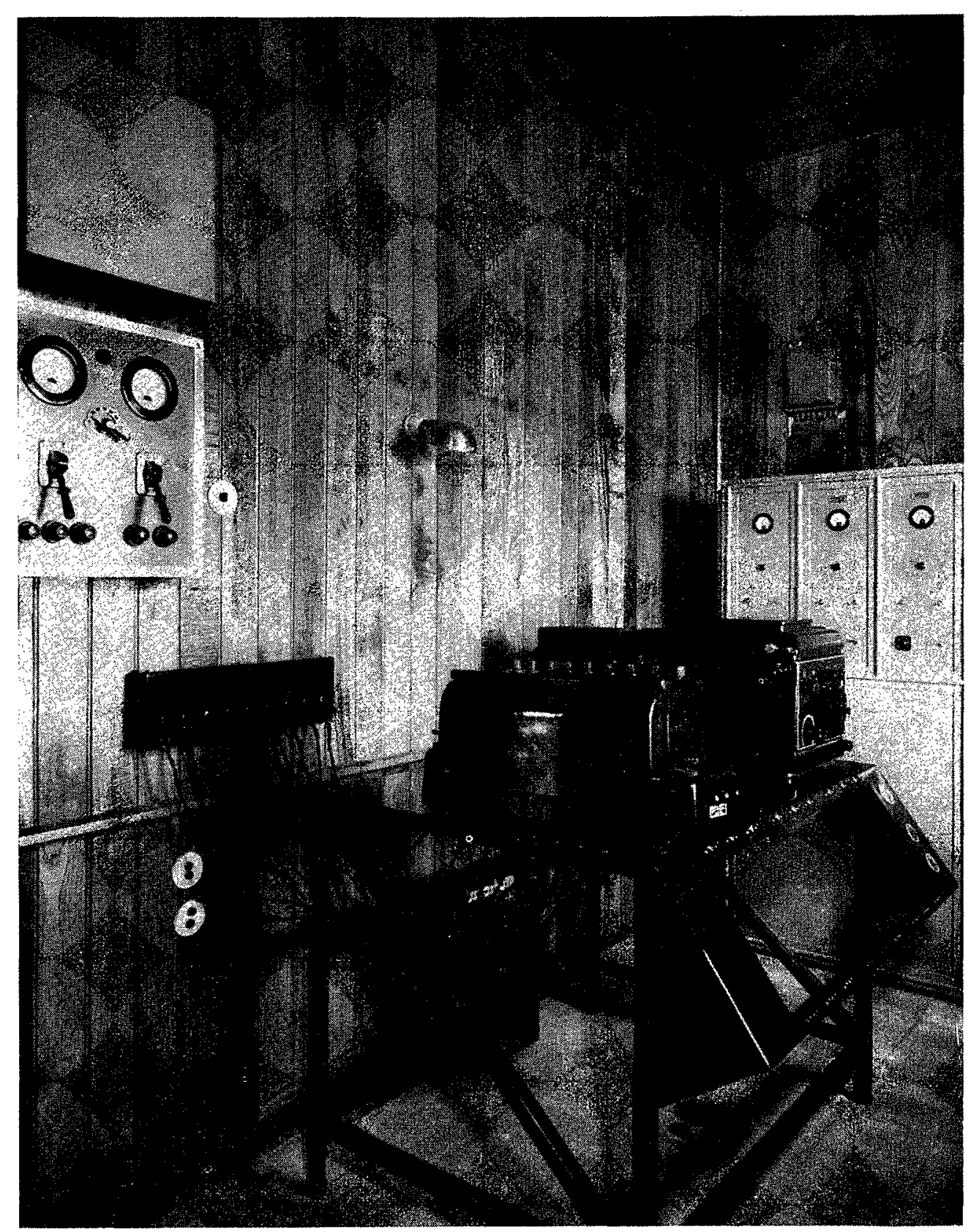

Pнoто C. - Oscillographe enregistreur des pressions.

$(+4,00),(0,00),(-2,35),(-4,35),(-6,45)$, $(-8,65),(-10,35)$.

De la mème façon, dans la paroi externe côté large, et en correspondance avec un second puits latéral, a été pratiquée une autre série d'ouvertures circulaires aux mêmes cotes, et une ouverture supplémentaire a été rajoutée à la cote $(+2,35)$. Lors des expériences qui furent faites à l'origine, les appareils cnregistreurs "Amsler », installés dans la seconde série d'ouvertures, ne donnèrent pas la preuve de leur aptitude à fomrnir une mesure précise de l'action dynamique de la houle, el c'est la raison pour laquelle ils ont élé supprimés dans la reconstruction actuelle. Les ouverlures de ce second puils qui onl élé fermées à celle occasion, pourront être utilisées pour l'installation éventuelle de nouveaux types d'appareillages, actuellement a l'étude.

Dans un troisieme puits, situé à l'intéricur par rapport aux deux premiers, ont été installés l'ascenseur, la pompe d'épuisement, ainsi qu'une 


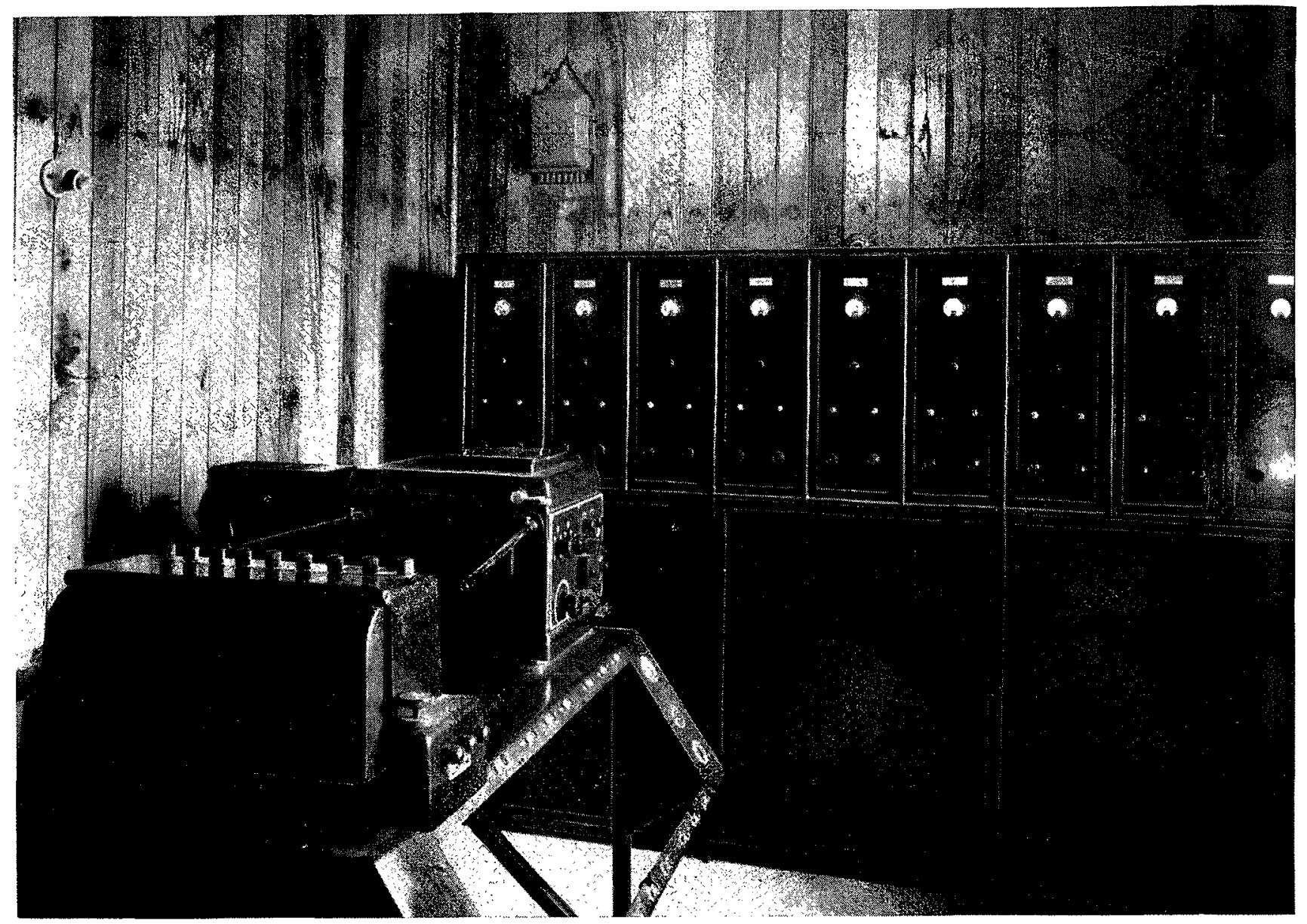

Photo D. - Statron pyonakénétrigue. Oscillographe et tableau de contròle des relèvements.

échelle de marin permettant d'atteindre le plancher de fondation du caisson, à environ $12 \mathrm{~m}$ au-dessous du niveau moyen de la mer.

Dans l'ouvrage d'avant-guerre, les appareils de mesure de pression, installés dans le premier puits aux diver'ses cotes indiquées, comportaient une plaque réceptrice mise en contact étroit avec: un cylindre de métal ferro-magnétique, autour duquel était enroulée une spirale conductrice parcourue par un courant électrique. La sollicitation exercée par la pression à mesurer faisait varier la capacité de magnétisation du petil cylindre et, par consćquent, la perméabilité du circuit magnétique et l'intensité du courant parcourant la spirale.

Celle-ci était insérée sur un des deux côtés d'un pont de mesure Maxwell qui comprenail en outre une inductance variable sur le coté adjacent, deux redresseurs sur les deux autres cotés, et, sur les deux diagonales, respectivement la source d'alimentation à courant alternatif à $5.000 \mathrm{~Hz}$, el l'équipage de mesure de l'appareil récepteur (oscillographe), précédé par un dispositif d'amplification.
Le récepteur étant branché et la mẽ̃ parfaitement calme, on mettait le pont à zéro au noyen de l'inductance variable. Et, cormme il a déja été indiqué, les variations de pression engendraient des variations proportionnelles d'inductance, qui déplaçaient l'équilibre du pont, et qui étaient transformées en variations de courant el enregistrées.

Par la suile, de nombreuses études et de nombreuses expériences ont été conduites en vue d'obtenir une transmission plus sûre et plus lacile des varialions de pression de la houle exercées sur le disque mćtallique en variations des paramètres des circuits électriques liés à l'oscillographe, et l'on est ainsi parvenu à l'appareillage fondamental suivant :

$1^{\circ}$ La placrue circulaire du récepteur a élé construite en acier harmonique : pour une épaisseur de 8/10 de millimètre, il s'est révélé capable d'offrir une sensibilité meilleure et une durće plus grande.

20 La plaque réceptrice des actions dynamiques de la houle, dont les déformations sont 


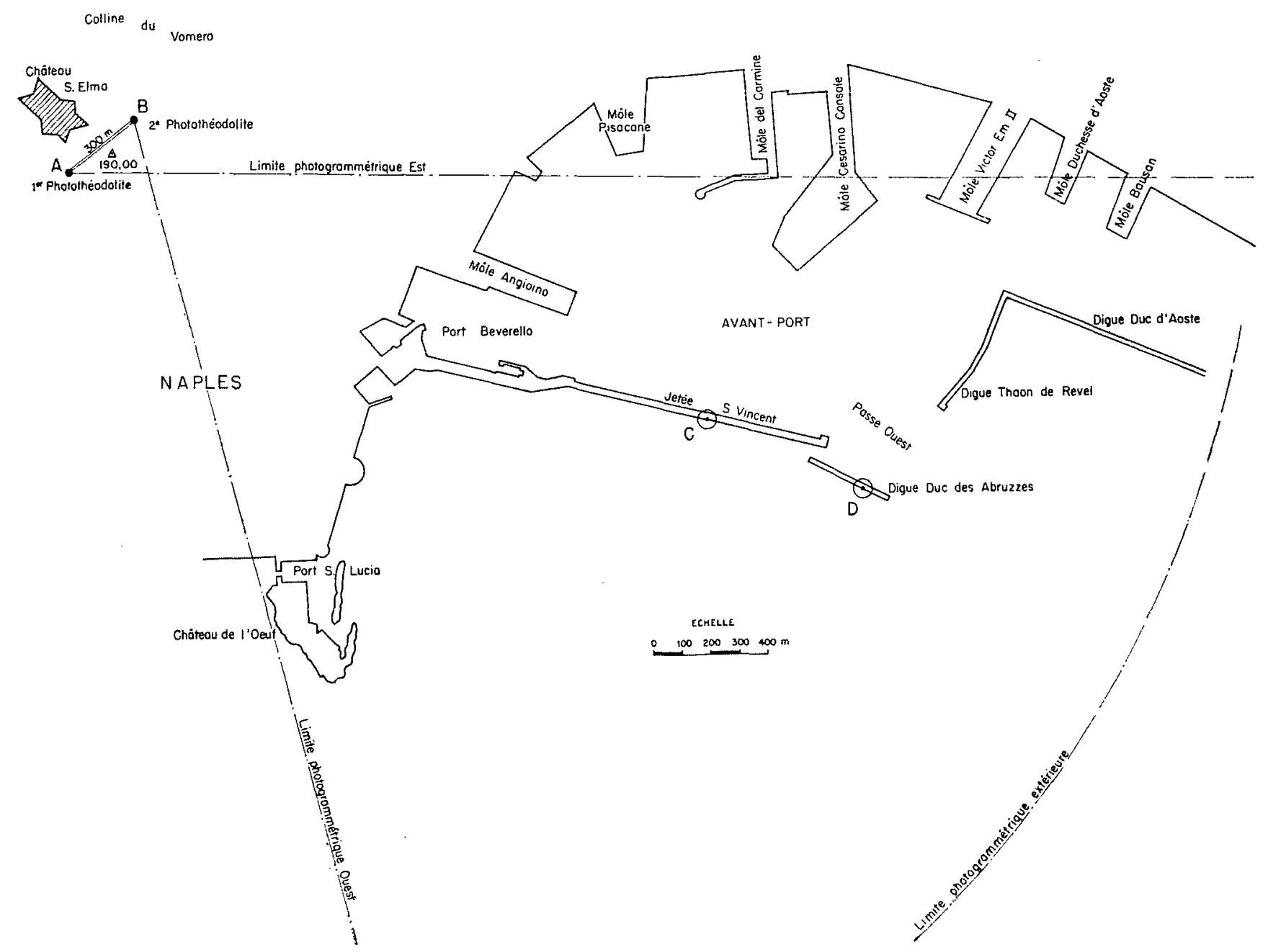

FIf. 1. - Plan d'ensemble des installations dynomaréométriques et stéréophotogrammétriques du port de Naples.
A, B : Stations stéréophotogrammétriques.
C: Cahine d'enregistrement dynomaréometrique.
D): Cabine de relèvements dynomaréométriques.
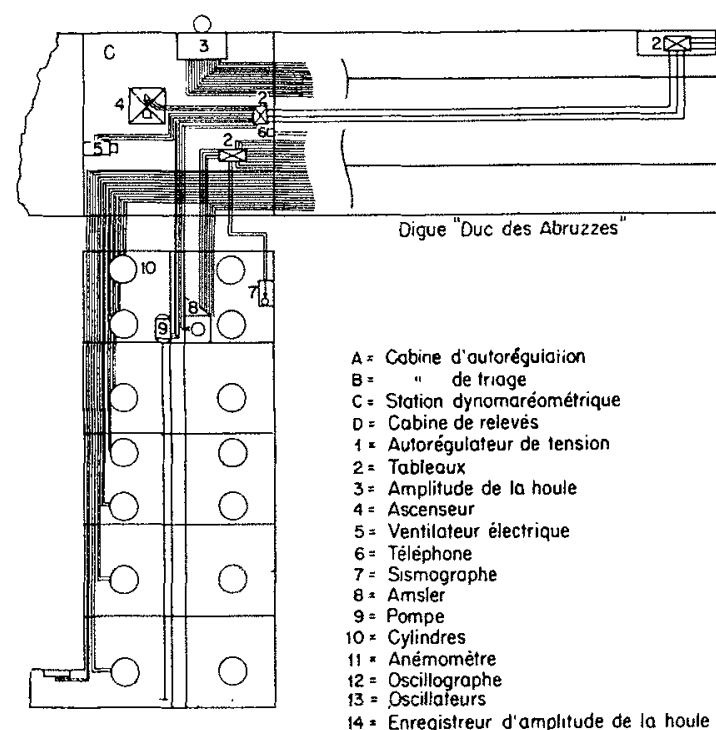

INSTALLATION ÉLECTRIQUE GÉNERALE

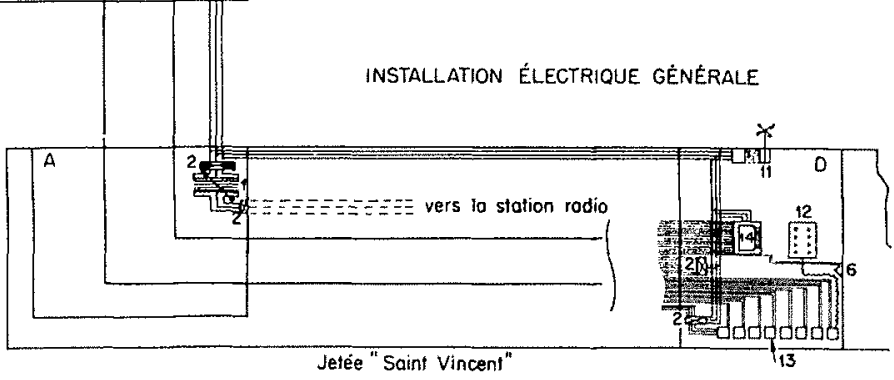

Fut 2 a. -.. Port on Narks. Station dynomaréométrique. 

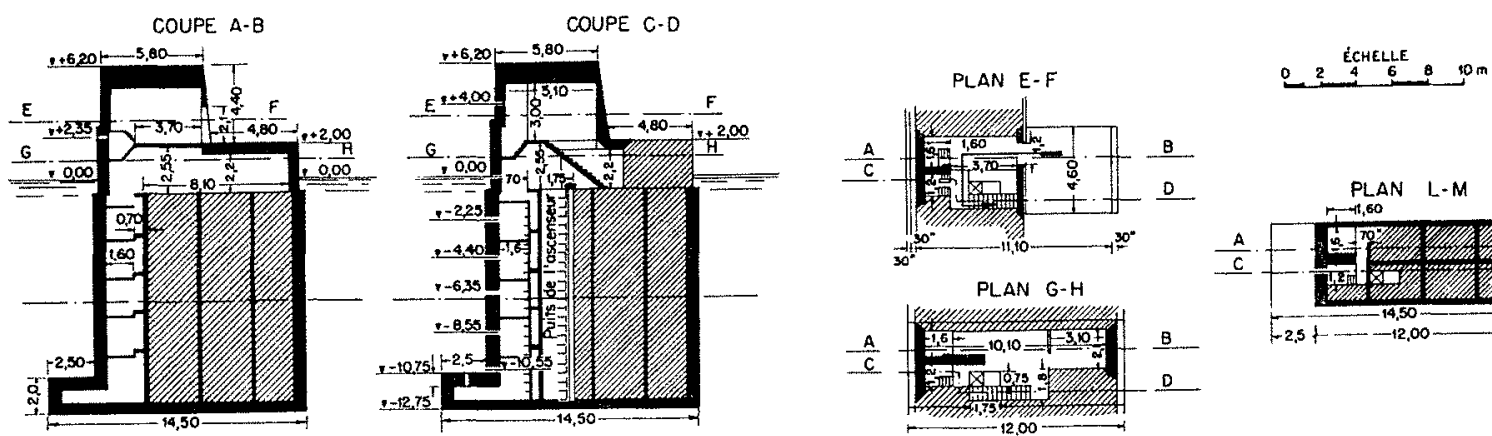

Fic. 2 b. - Pont de Naples. Caisson de réception « Digue Duc des Abruzzes 》.

transmises par le jeu d'un levier décuplicateur à un noyau de fer carbonyle qui se déplace dans le champ magnétique d'une inductance.

Les variations des efforts dynamiques de la mer exercés sur le capteur conduisent à des variations de fréquence d'un oscillateur ayant normalement une fréquence de $600.000 \mathrm{~Hz}$.

La variation de fréquence, due à la variation

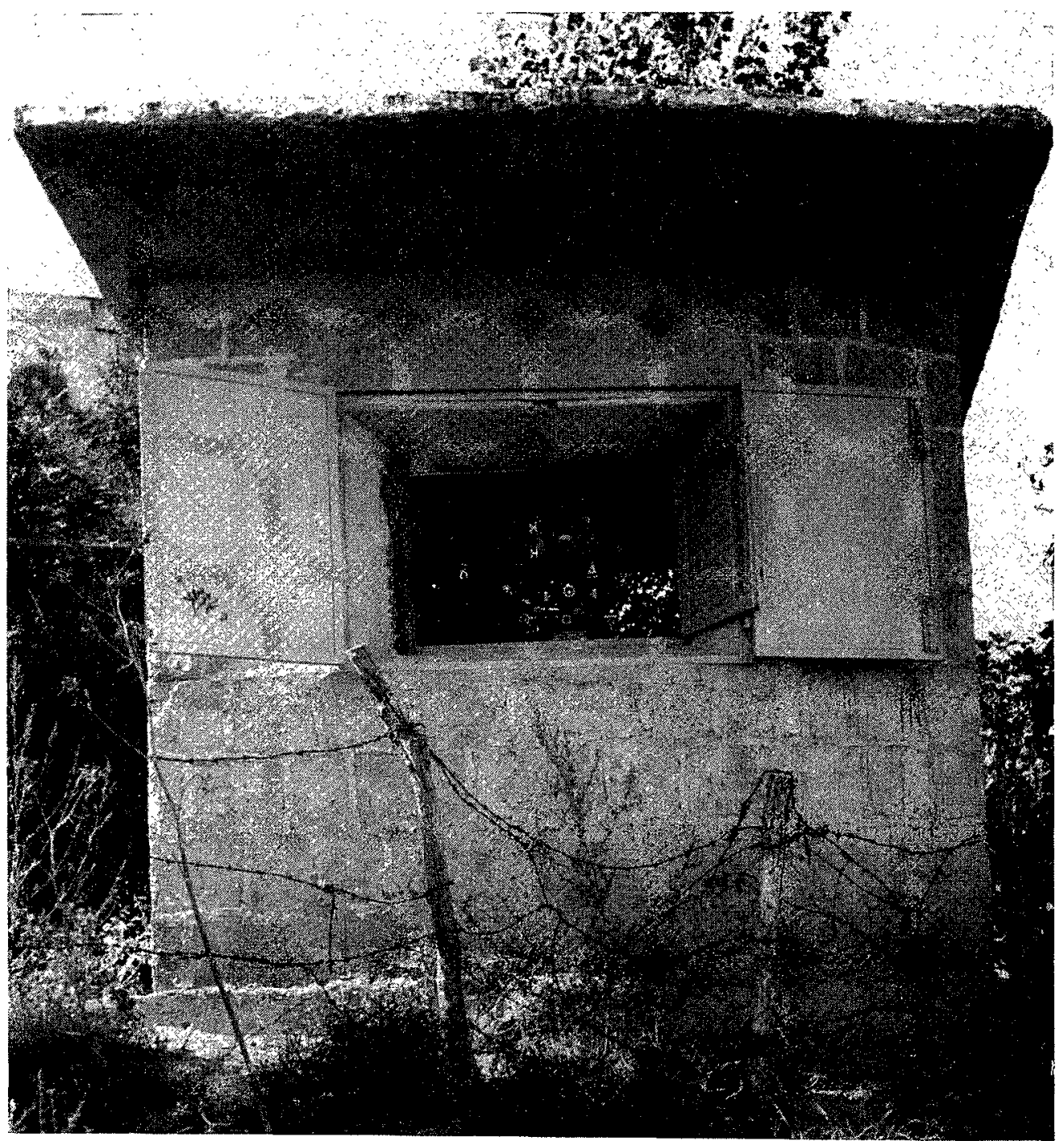

Photo E. - Station Photogramiétrigue de Naples. Vue extéricure d'um appareil photographique (cabine B). 


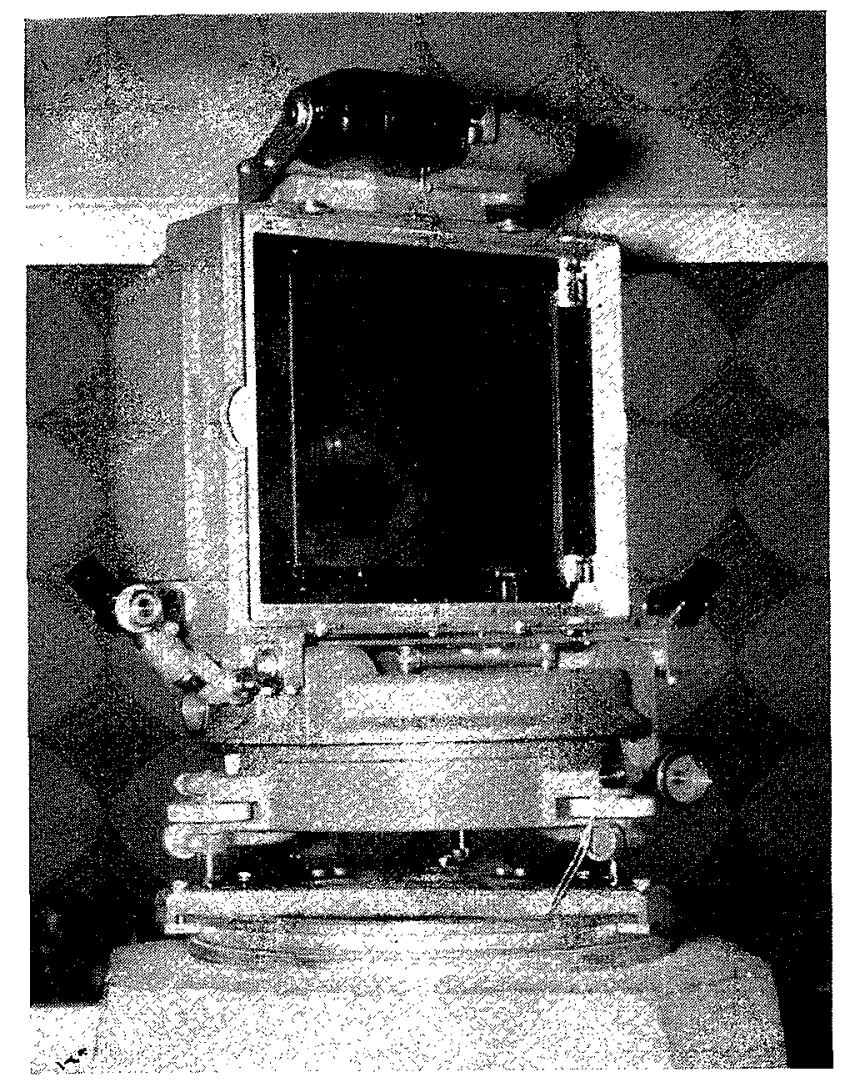

Photo $F$. - Station photogrammétrique de Nalites. Vue postéricure d'un appareil photographique.

de pression, à la sortic de l'oscillateur, produit un courant qui est redressé puis envoyé à l'oscillographe.

Il convient de noter que l'on peut également obtenir l'enregistrement oscillographique des pressions en remplaçant les variations de fríquence par des variations de résistance.

3 "Il a ćté procédé au blindage des appareils comportant des tubes électroniques, de façon à les protéger contre l'influence du milieu marin, spécialement contre l'humidité salée.

Les perfectionnements ainsi introduits ont permis de bénéficier des avantages suivants :

a) simplification de l'ensemble de l'appareillage;

b) efficacité de la substitution, par un noyau de fer carbonyle, du fer en lamelles qui s'oxydait rapidement;

c) plus grande sensihilité de toute l'installation.

\section{Station stéréophotogrammétrique}

Cette station est le complément indispensable de la station dynomariométrique, en ce qu'elle csl en mesure de fournir, au moyen de relevés stérćophotogrammétriques, les caractéristiques morphologicques de la houle, et leurs modifica- tions à l'approche des ouvrages, toul ceci en synchronisme avec l'enregistrement des pressions.

Après quelques expériences préliminaires, au moven d'appareils qui n'étaient pas adaptés à cetle recherche, il fut procédé à l'étude et à l'exécution de l'installation définitive, désormais mise au point et se présentant ainsi qu'il suit :

$1^{\circ}$ Les deux postes sont situés sur les collines de Saint-Martin, à environ $190 \mathrm{~m}$ au-dessus du niveau de la mer et à une distance horizontale de la surface de l'eau d'environ $1000 \mathrm{~m}$; elles sont distantes mutuellement d'environ $300 \mathrm{~m}$ (fig. 1). Dans chaque poste est installé un phototheodolite, qui occupe une cabine approprice construite en maconnerie.

$2^{\circ}$ Le photothéodolite comporte essentiellement une chambre photographique munie d'an objectil de $215 \mathrm{~mm}$ de longueur totale; le format des images est de $180 \mathrm{~mm}$ sur $200 \mathrm{~mm}$.

Le magasin porte-films peut contenir $60 \mathrm{~m}$ de pellicule, ce qui permet d'effectuer environ 300 photogrammes. Un petit moteur électrique, relié a l'appareil par un dispositif approprié, provoque le déroulement du film et l'armement de l'obturateur dans un laps de temps de 6 secondes environ.

L'ouverture des deux photothéodolites est obtenue avec un parfait synchronisme au moyen d'une commande électrigue à distance qui, dans le cas présent, se trouve dans la cabine même de commande de la station dynomaréométrifue, à la jetće Saint-Vincent.

Pour faciliter les préparalifs des expériences, une liaison téléphonique a été mise en placo entre les cabines phologrammétriques et la station dynomaréométrique.

$3^{\circ}$ Sur chaque phologramme recueilli, sont enregistrés automatiquement le numéro de Ja chambre, la Iongueur focale, le numéro d'ordre. du photogramme et l'heure de la photographie. Ces dernic̀res indications chronologiques sont particulièrement utiles pour faire correspondre les photogrammes avec les enregistrements dynomarcométriques, Ils peuvent servir également à confirmer la valeur de la vitesse de propagation de la houle obtenue par d'autres procédés.

En relevant, à de tris hrefs intervalles, la surface de la mer agitée, il sera désormais possible de suive les avatars de la tempête.

$4^{\circ}$ Pour restituer les tirages des photogrammes simultanés, il a ćté prévu d'utiliser des appareils de restitution normaux que possedent presque lous les pays, dans un certain nombre de types. C'est pourquoi la longueur focale el le format des imases ont ité choisis en accord 


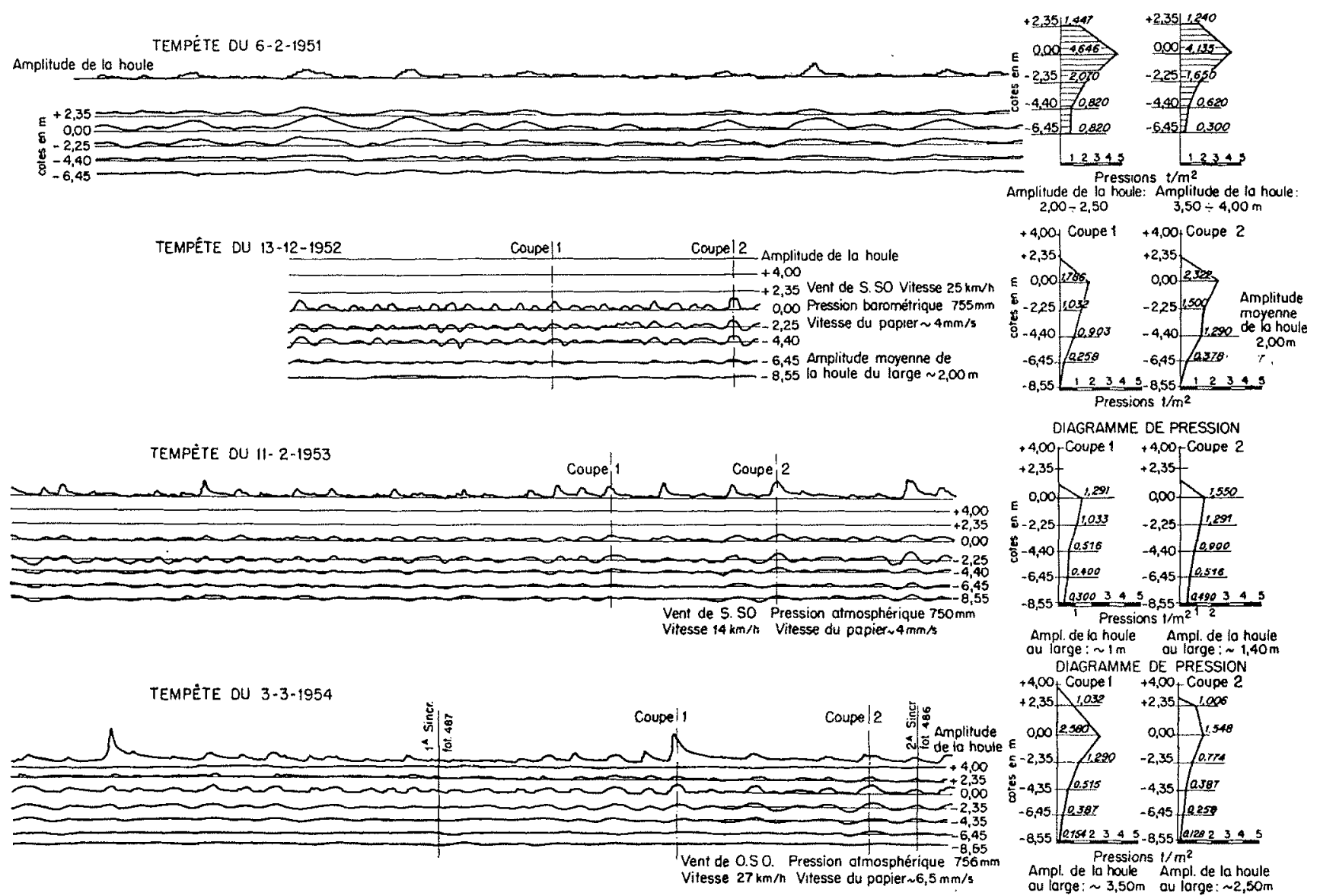

Fig. $3 a$. - Diagrammes oscillographiques des pressions de la houle

et diagrammes des pressions maxima. - Relèvements dynamométriques.

avec les caractéristiques des instruments de restitution.

Bien que ces instruments eussent permis une plus grande liberté de prise de vues, nous avons préféré maintenir horizontaux les axes de nos photothéodolites, de façon à pouvoir éventuellement faire des mesures stéréogrammétriques à l'aide de simples stéréocomparateurs.

$5^{\circ}$ Le champ d'action de la station stéréophotogrammétrique, qui est d'environ $4 \mathrm{~km}$, se révèle suffisant pour nos études.

A titre d'exemple, voici quelques diagrammes des pressions de la houle (fig. $3 a$ ) releves au cours des tempêtes des années 1951, 1952, 1953 et 1954 , et quelques relevés stéréophotogrammétriques des tempêtes de 1951 et 1954 (Diag. I et II) où l'on dis!ingue très bien le plan de la houle du large et celui de la houle d'expansion dans la zone de l'avant-port.

En poursuivant des études et des expériences déjà entreprises systématiquement et avec grand soin, nous comptons pouvoir parvenir à préciser le régime hydrodynamique du golfe de Naples, et à fournir d'autre part le modèle d'une station dynomaréométrique et stéréophotogrammétrique qui a déjà suscité des approbations encourageantes de la part du * Comité international pour l'étude des efforts dus aux lames », lors des réunions de Paris, de Naples et de Hanovre.

Ainsi que disait Baudelatre dans une des poésies les plus expressives des Fleurs du Mal:

Homme libre, toujours tu chériras la mer,

La mer est ton miroir : tu contemples ton âme Dans le déroulement infini de sa lame,

Et ton esprit n'est pas un goufre moins amer.

Vous êtes tous les deux ténébreux et discrets

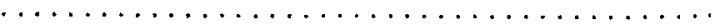

Tant vous êtes jaloux de garder vos secrets!

Alors que les vicissitudes de la vie nous prouvent combien l'âme humaine est vraiment insondable, les océanographes, dans leurs études sévères, ininterrompues et passionnées, s'efforcent, non sans de féconds résultats, à pénétrer les mystères de la mer dans son dynamisme et dans la vie miraculeuse qui s'accomplit de par l'immensité de son essence, seule réalité offranl l'idée de l'infini dans le temps et dans l'espace. 
LUIGI GRECO: Aperçu sur les théories mathématiques de la houle, et nécessité de recherches ex

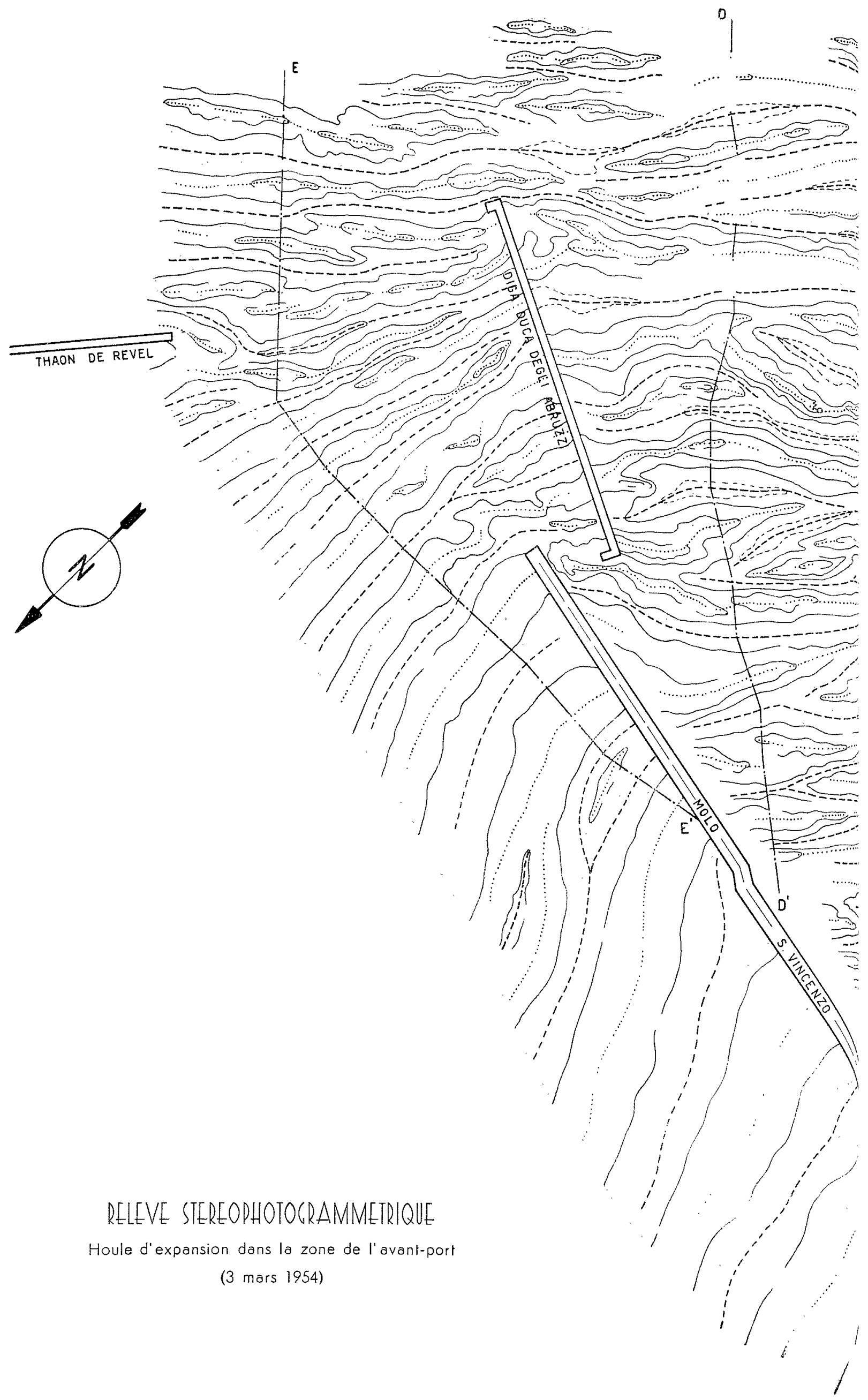




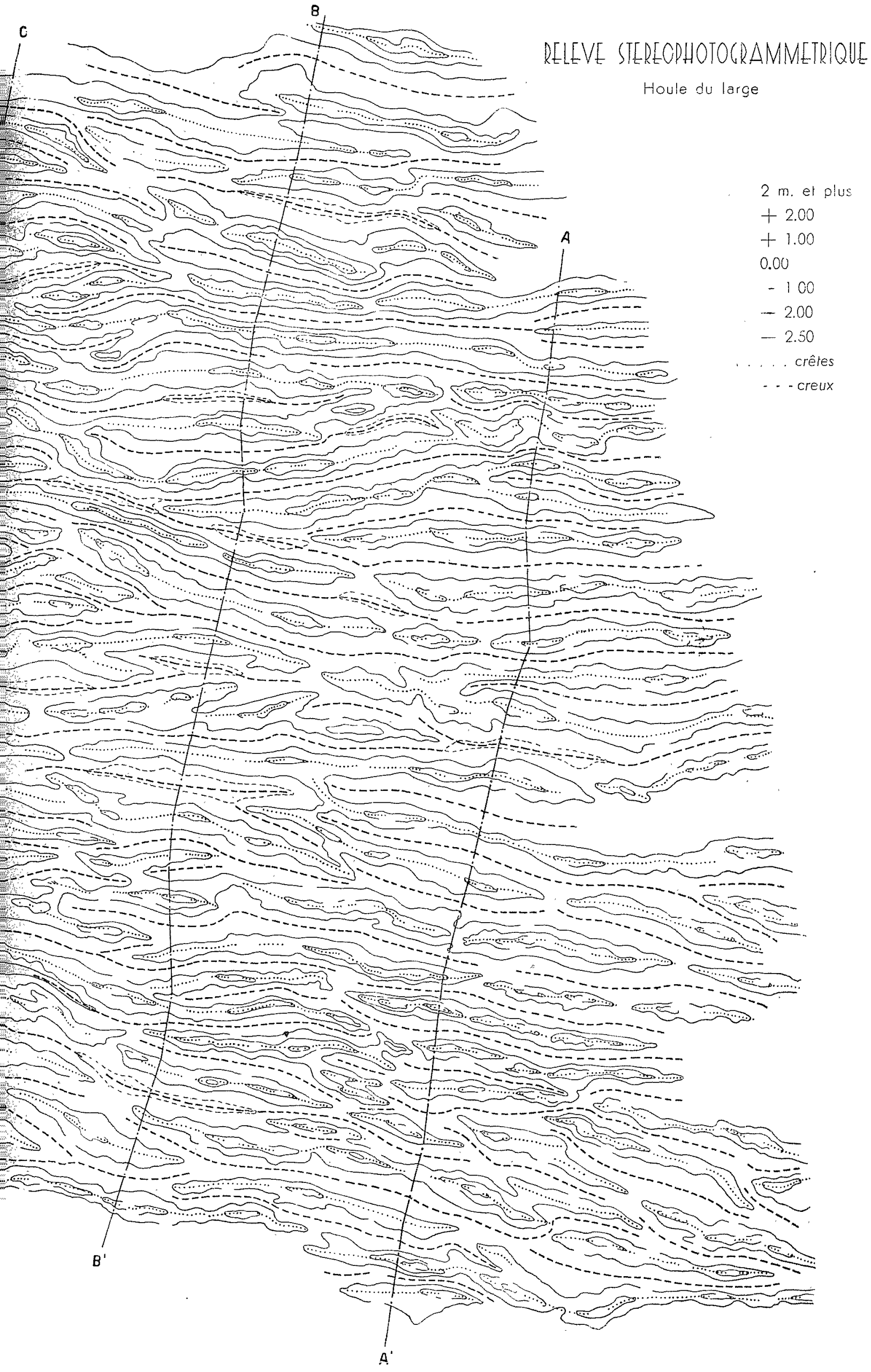


:

$: A$ A

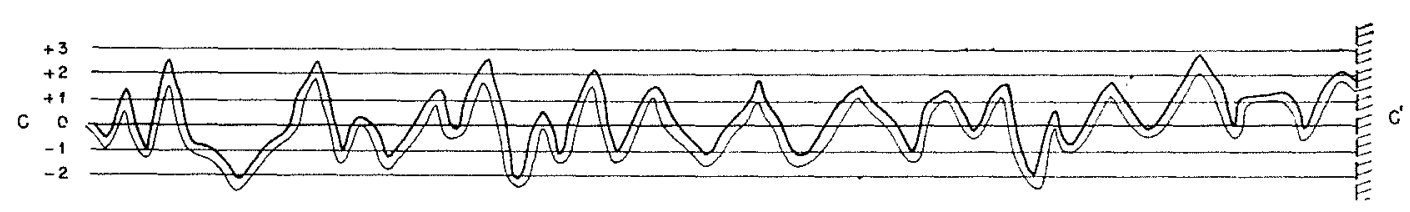

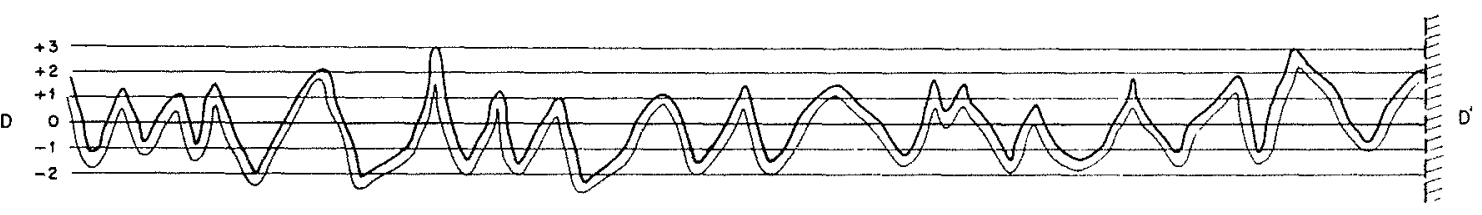

\

W

UA

$\checkmark$.

FIG. 3 b. - Fragments du relevé de la houle au large.
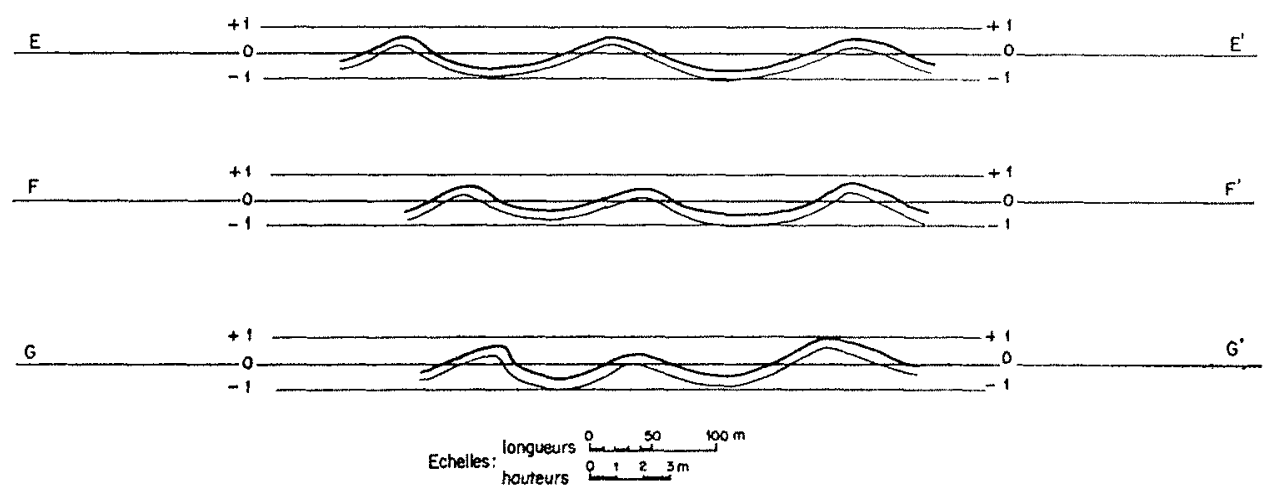

Fia. 3 c. - Fragments du relevé de la houle d'expansion (12 novembre 1951). 

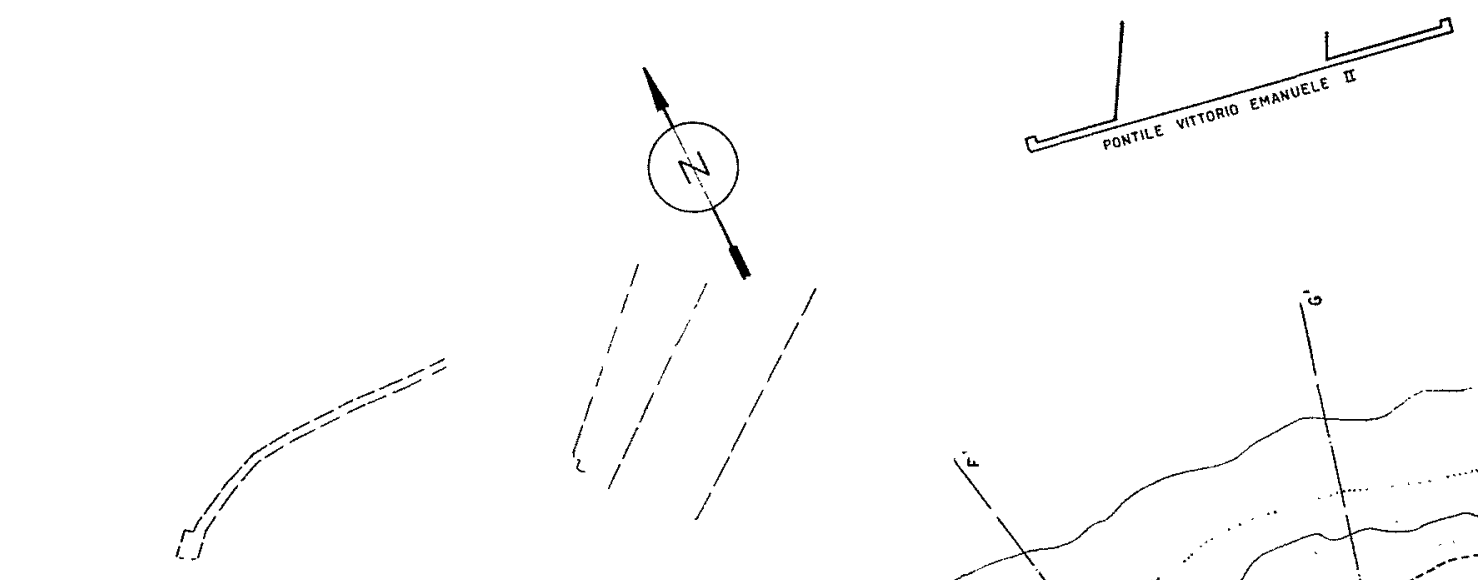

RELLVE STEREODHOTOCRAMMLTRIQUE Houle d'expansion dans la zone de l'avant-port

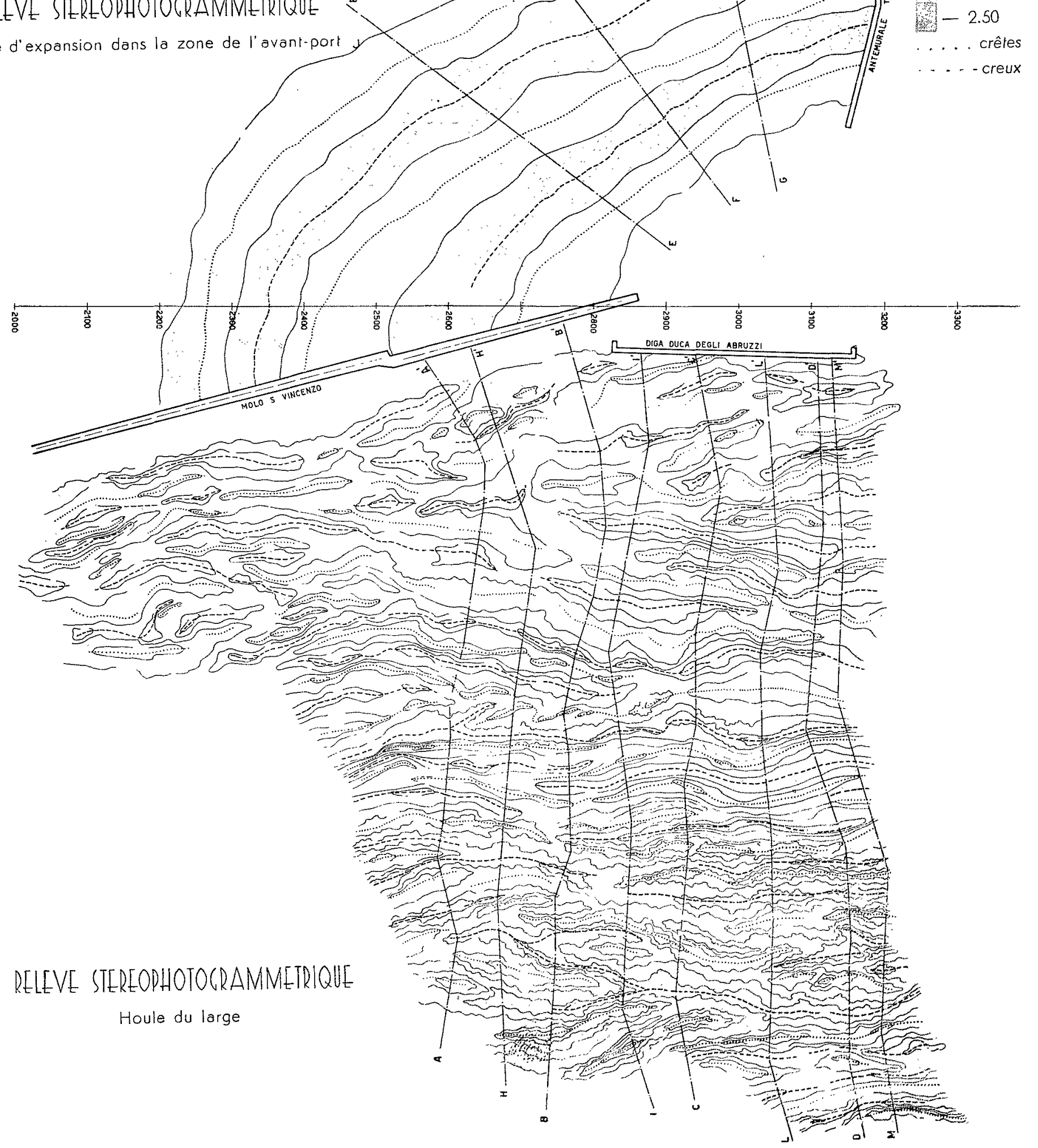


DIAG. II
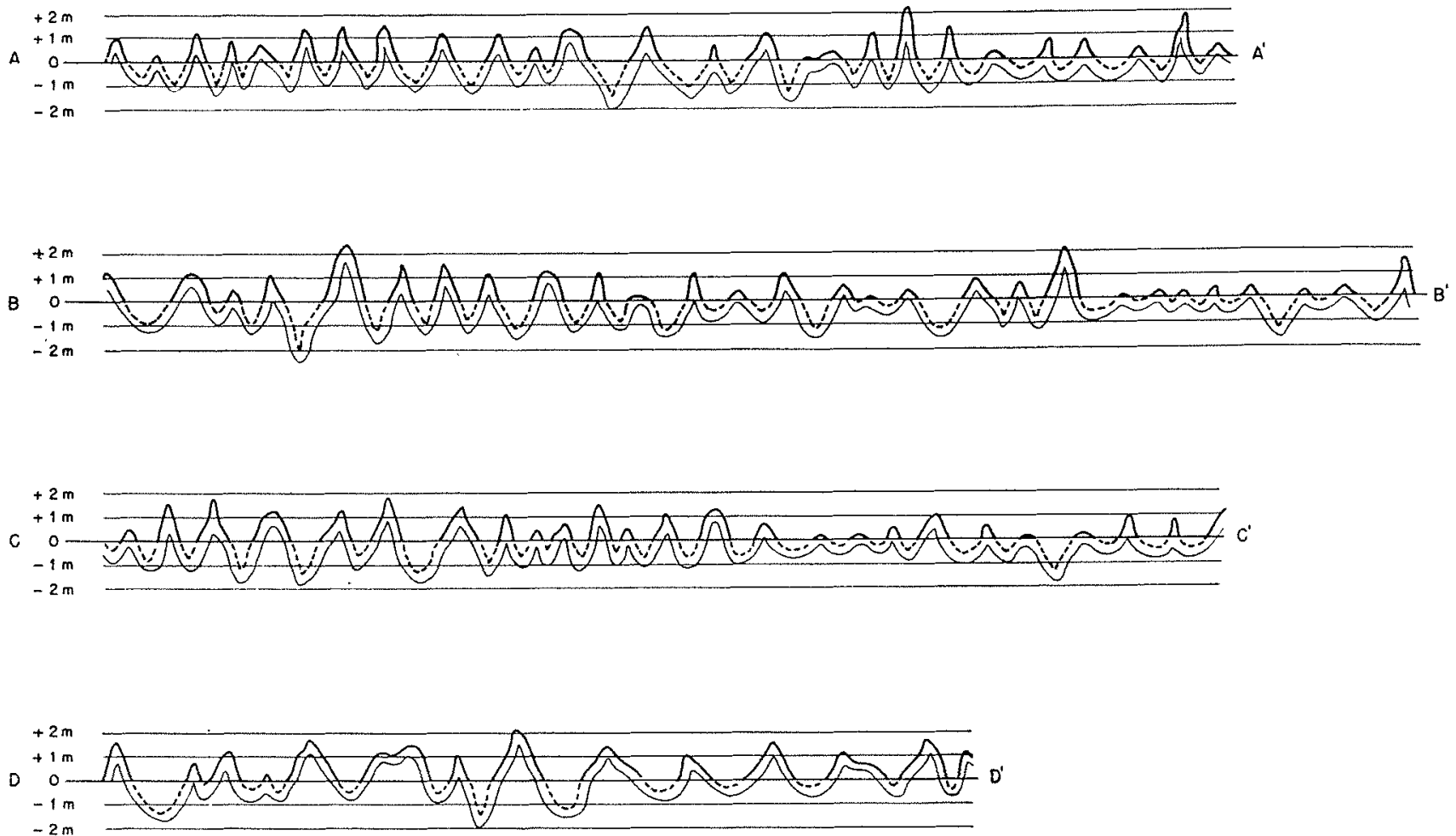

Fragments du relevé de la houle au large.

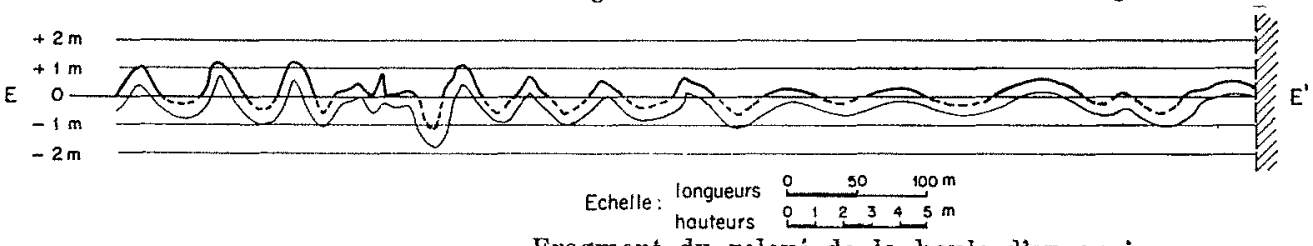

Fragment du relevé de la houle d'expansion.

\section{BIBLIOGRAPHIE}

(1) Leonardo DA Vinci : Del moto e misura dell'acqua, a cura di E. Carusi ed A. Favaro. Bologna, Zanichelli, 1923, Libro I.

(2) LeONardo Da Vincl: Delle cose portate dal'acqua, Libro VII.

(3) Agatino D'Arrigo : Ricerche sul regime dei litorali del Mediterraneo, a cura del Consiglio Nazionale delle Ricerche, Roma, 1936. - Sulle fasi di regime del litorale deltizio del Tevere. « Annali dei Lavori Pubblici 》fasc. $3^{\circ}$, Roma, 1932. - Ricerche sulla linea neutra e sull'evoluzione morfolitologica della piattaforma litorale inerente al Golfo di Palermo. «Atti della SIPS, XXIV Riunione », Palermo, 1935. - Ricerche sulla linea neutra inerente al Golfo di Gaeta e sul regime del litorale deltizio del Vol turno. "Annali dei Lavori Pubblici », fasc. $2^{\circ}$, Roma, 1934.

(4) Paolo Cornaglia : Sul regime delle spiagge e sulla regolazione dei porti. Torino, Stamperia Reale della ditta G. B. Paravia e Comp., 1891.

(5) Luigi Greco : La nuova opera a difesa della bocca del porto di Napoli con infrastruttura di massi artificiali ciclopici. «Annali dei Lavori Pubblici », fasc. $12^{\circ}$, Roma, 1926.

(6) Franz von Gerstner : Abhandlungen der Kömigl. Böhmischen Gesellschaft der Wissenschaft.
(7) Sir Geonge AIry : Enciclopaedia Metropolitana.

(8) M. BoussinesQ : Annales des Ponts et Chaussées, $1888,1^{\text {er }}$ semestre.

(9) D. Galllard : Waves action in relation to Engineering Structures. "Professional Papers of the Corps of Engineers U. S. Army », Washington, 1904.

(10) Luigi Greco : La nouvelle digue à l'entrée du port de Naples. Istituto Arti Grafiche Rispoli, Via Diodato Lioy, 14, Napoli, 1931.

(11) Henry F Fre : Le problème des vagues. "Schweizerische Bauzeitung 》, vol. 108 , n. 1 et 2 , juillet 1936 .

(12) H. Bouasse : Houles, rides, seiches et marées. Paris, Librairie Delagrave, Rue Soufflot, 15, 1924.

(13) Luigr Greco : Impianti sperimentali per lo studio del moto ondoso nel porto di Napoli. « Annali dell'Istituto Superiore Navale », vol. IV, fase. $2^{\circ}$, 1935.

(14) Ariserto Albertazzi : Recenti esperienze sulle azioni dinamiche delle onde contro le opere marittime. « Annali dei Lavori Pubblici », Roma, 1932. - Sarvatora Levi : L'impianto realizzato nel porto di Genova per la misura del moto ondoso. « Annali dei Lavori Pubblici », Roma, 1933. - Pressioni esercitate dal mare contro le dighe a parete verticale. « L'Ingegnere 》, Roma, 1934. 